\title{
Projeto, Construção e Controle de um Chopper Óptico operando na faixa de $100 \mathrm{~Hz}-300 \mathrm{~Hz}$
}

\author{
Carlos Junior Felix Rodrigues* Germano Maioli Penello** \\ Andrei Giordano Holanda Battistel ${ }^{* * *}$ \\ * Departmento de Engenharia Eletrônica e de Computação, \\ Universidade Federal do Rio de Janeiro, RJ, (e-mail: \\ carlosrodrigues@poli.ufrj.br). \\ ** Instituto de Física, Universidade Federal do Rio de Janeiro, RJ, \\ (e-mail: gpenello@if.ufrj.br). \\ *** Departamento de Eletrônica e Telecomunicaçôes, Univeridade do \\ Estado do Rio de Janeiro, RJ (e-mail: andrei.battistel@gmail.com )
}

\begin{abstract}
This project consists in the fabrication of an optical chopper and the development of the control method used for its operation. The system identification of a control constitutes the most important part for the PID parameters optimization. One of the characteristics of this technique is that it is not necessary to know all the information about the system. The main motivation of this article is to optimize the control of an optical chopper for its utilization in a high precision optical measurements system. In this article, we decided to optimize the control through the generalization of its behavior through an algorithm that understands how a system responds to a specific stimuli and using Genetic Algorithm.

Resumo: Este projeto consiste na criação de um chopper óptico e o método de controle utilizado para seu funcionamento. A identificação de um sistema de controle constitui parte importante para a otimização dos parâmetros PID. Uma das características desta técnica é que não se faz necessário conhecer todas as informações acerca do sistema. A motivação principal deste artigo é otimizar um controle da rotação de um disco de um chopper óptico para sua utilização num sistema de medidas ópticas de alta precisão. Neste artigo, decidimos otimizar seu controle por meio da generalização do seu comportamento através de um algoritmo que compreende como um sistema responde à estimulos e utilizando Algoritmo Genético.
\end{abstract}

Keywords: Optical Devices, PID, Arduino, Frequency Control, Genetic Algorithm

Palavras-chaves: Dispositivos Ópticos, PID, Arduino, Controle de Frequência, Algoritmo Genético

\section{INTRODUÇÃO}

O Chopper óptico é um instrumento científico utilizado com a finalidade de modular a intensidade de um feixe de luz, o que é feito interrompendo a luz periodicamente através de um disco dentado. Tal equipamento é extremamente importante para investigação das propriedades elétricas e ópticas de materiais semicondutores nanoestruturados e de dispositivos optoeletrônicos(M. Penello et al. (2019)). Para seu perfeito funcionamento, é necessário que o disco dentado se mantenha girando em uma frequência fixa especificada por seu utilizador. Alguns obstáculos devem ser superados para obter uma boa precisão nesta operação. Além da interferência de diversos fatores externos ao sistema, há também dificuldades impostas pela própria configuração de montagem do dispositivo. São exemplos a geometria ou material da roda e suas estruturas de

\footnotetext{
* Suporte financeiro da pesquisa oferecido pela Fundação Carlos Chagas de Amparo à Pesquisa do Estado do Rio de Janeiro(FAPERJ).
}

suporte, fatores estes que interferem significativamente no funcionamento do chopper.

O modelo da empresa SRS é um exemplo de chopper óptico muito utilizado na caracterização de fotodetectores de infravermelho e tem seu custo em torno de U\$ 2.000,00, incluindo custos de manuseio e envio para o Brasil. No entanto, o chopper é um equipamento que pode ser construído por um preço significativamente mais acessível utilizando ferramentas e dispositivos de fácil obtenção. Desta forma, a motivação deste trabalho se dá no sentido de suprir a necessidade deste importante equipamento em laboratório, bem como na possibilidade de se criar uma alternativa de baixo custo para utilização em pesquisas relacionadas.

No presente trabalho, utilizou-se uma roda dentada de $10,25 \mathrm{~cm}$ de diâmetro com 6 furos impressa por uma impressora 3D para construção do Chopper. Esta roda encontra-se acoplada a um motor DC que recebe uma corrente através de uma fonte fonte chaveada controlada pelo sinal de PWM (Pulse Width Modulation) de um 
Arduino. Através do valor do ciclo de trabalho do PWM é controlada a frequência em que a roda gira. Para realizar o controle do sistema, foi desenvolvido um circuito sensor que fornece um valor lógico 1 ou 0 quando houver, respectivamente, passagem de luz ou não pelo furo da roda dentada. Disposto desses dados, o código no Arduino determina a frequência da roda. Tais características (tamanho, material, tipo de motor, etc) são influenciadores em como o sistema responderá e qual controle será o mais otimizado.

A fim de controlar a velocidade de rotação da roda, é necessário adequar o sistema à inúmeras variáveis, algumas delas difíceis de serem modeladas. Neste trabalho foi utilizada a identificação utilizando uma abordagem entrada-saída com um sinal binário pseudo-randômico (PRBS)(Fairweather et al. (2011)) na entrada. De posse de uma descrição matemática do sistema, é possível utilizar rotinas de otimização dos parâmetros do controle. Neste trabalho, optamos por um controlador do tipo PID (Dorf (2001)) ajustado utilizando uma técnica de algoritmo genético visando minimizar o erro.

\section{CARACTERÍSTICAS DO CHOPPER}

O desenho 3D da roda contendo seis furos é demonstrado na Figura 1. A impressão 3D foi realizada utilizando filamento ABS, um tipo de material plástico de alta resistência mecânica e térmica(Farbman and McCoy (2016)). Para medir a frequência da roda, é utilizado um sensor com fototransistor (TCST1230) localizado abaixo da roda dentada.

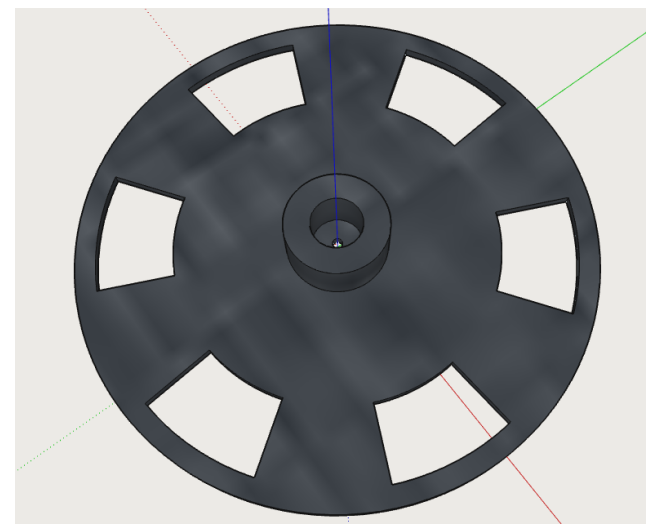

Figura 1. Roda perfurada do Chopper Óptico.

Acoplado à roda está o motor de corrente contínua de 5 volts que irá fazer a rotação do disco dentado. O motor é constituído de um eixo conectado ao rotor, que é a parte girante do motor. Para garantir uma maior estabilidade o motor se encontra fixado por uma alça ao suporte, ambas feitas em impressão 3D.

\subsection{Circuito do Sensor}

Foi projetado um circuito eletrônico que serve como sensor para se obter o valor da frequência em que a roda está operando. A saída do sensor óptico é ligada a um amplificador operacional funcionando como um circuito comparador. Quando a tensão da entrada inversora do amplificador operacional está acima de $1 \mathrm{~V}$ (tensão definida pelo divisor
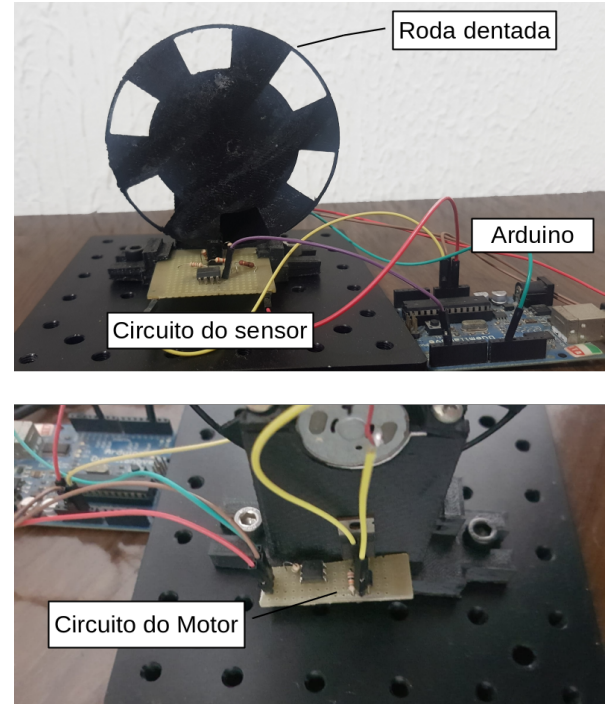

Figura 2. Foto do chopper com elementos destacados.

de tensão entre R3 e R4) o valor da saída satura em 3,7 V. Quando a tensão da entrada inversora é menor do que $1 \mathrm{~V}$, obtem-se 0V na saída.

O sensor TCST1230 funciona de forma que, ao receber luz, a tensão em R2 valerá $1,56 \mathrm{~V}$ e sem luz $342 \mathrm{mV}$, valores que condizem em como o amplificador operacional está configurado. Sendo assim, a saída entrará no pino digital do Arduino que lerá o valor de $3,7 \mathrm{~V}$ como um valor lógico 1 e $0 \mathrm{~V}$ como um valor lógico 0 .

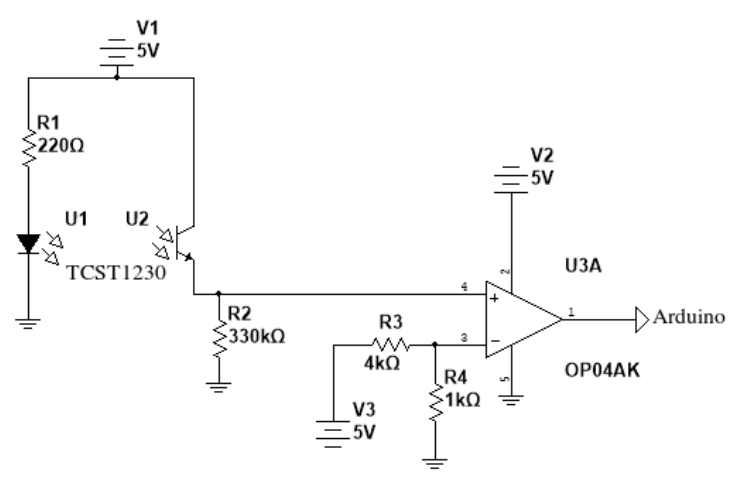

Figura 3. Sistema eletrônico do sensor da frequência da roda.

\subsection{Circuito de Controle do Motor}

Para que o controle funcionasse corretamente, foi projetado um circuito eletrônico que fosse capaz de manipular a rotação do motor. Além de controlar o motor, o circuito também tem o objetivo de isolar o Arduino (que é responsável pela parte lógica) do sistema do circuito, para isso foi usado um optoacoplador.

A Figura 4 mostra o circuito eletrônico utilizado para realizar o controle de corrente no motor DC. Ao lado direito do optoacoplador está projetado o circuito de chaveamento do motor com MOSFET. 


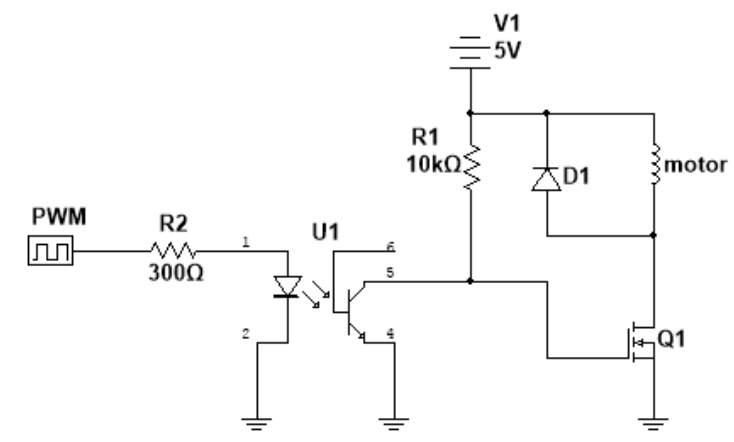

Figura 4. Sistema eletrônico de controle do motor.

\section{MODELAGEM MATEMÁTICA DO SISTEMA}

Para o projeto do controle da frequência do Chopper, optou-se pela utilização de um modelo identificado como ponto de partida. Tal escolha deve-se a dificuldade de obter os valores de certos parâmetros, como o momento de inércia do disco; além da presença de incertezas, como o caso dos componentes eletrônicos. O objetivo deste procedimento é a obtenção de um modelo a partir do qual o controlador PID possa ser sintonizado por um algoritmo genético em ambiente de simulação.

Os sinais de entrada escolhidos são do tipo PRBS, visando minimizar os efeitos do ruído presente no sistema como também excitar o sistema em toda a faixa de frequências de interesse. Os dados do ensaio foram divididos em dois conjuntos, dos quais um é empregado para a identificação e o outro para a validação (Aguirre, 2007). A validação consiste em comparar a saída medida y com uma saída simulada do modelo $\hat{y}$ para a entrada utilizada no ensaio. Após a estimativa de diversos modelos lineares, optou-se pelo modelo da Eq. (1).

$$
G(s)=\frac{-(0.7004 s+0.03438)}{\left(s^{2}+0.5683 s+0.01888\right)}
$$

O critério empregado para a escolha do modelo deu-se pelo menor erro quadrático, $e=(y-\hat{y})^{2}$, entre os modelos obtidos. A comparação entre as saídas medida e simulada pode ser vista na Fig. 5, mostrando que o resultado obtido é bastante razoável. É interessante notar que este sistema possui um ganho DC negativo, uma vez que a corrente de entrada diminui conforme o PWM aumenta em função do resistor pull up que foi colocado para realizar o chaveamento do transistor MOSFET.

\section{ALGORITMO GENÉTICO}

Algoritmos Genéticos são uma forma de achar soluções para problemas de otimização que se baseiam na natureza biológica da evolução cujo preceito definidor é a sobrevivência do mais apto. Seu resultado consiste em uma população de soluções selecionada por uma função avaliadora. Tal técnica se encontra bastante útil para a resolução de problemas onde há valores que precisam ser determinados, como o caso das constantes do controlador PID(Zhang et al. (2009)), servindo de alternativa ao método ZieglerNichols(F. M. Amaral (2009)), que nada mais é do que um ajuste fino posterior a lances iniciais.

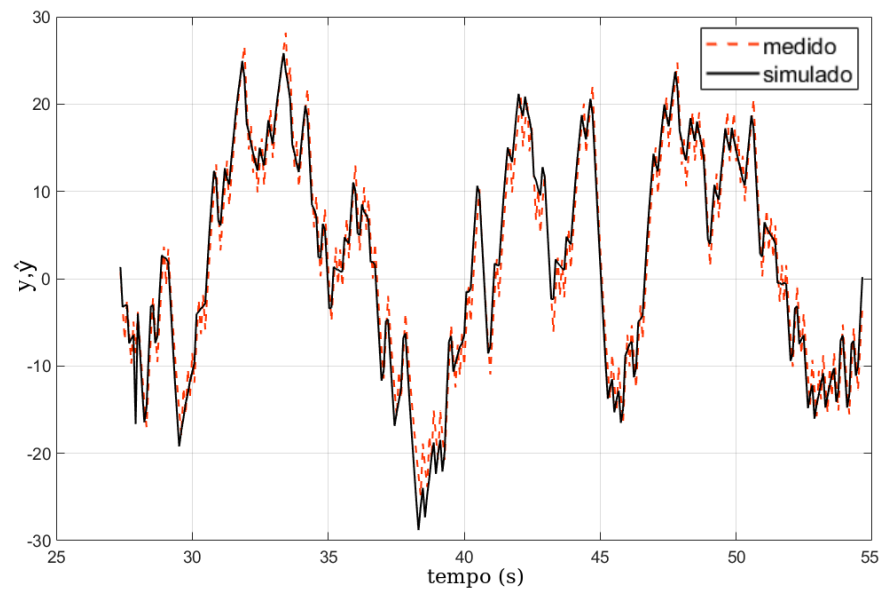

Figura 5. Validação do modelo identificado.

Dentro deste método temos uma população de indivíduos que representam possíveis soluções para o problema. Essa população interage entre si e é capaz de gerar indivíduos cada vez mais aptos com base nas operações de seleção, cruzamento e mutação. A seleção é realizada com base em uma função avaliadora (fitness function) que irá definir quais indivíduos possuem maior oportunidade para reproduzir. Já a mutação tem como objetivo garantir a variabilidade entre indivíduos. Para este trabalho, definimos que a seleção seria feita pelo método da roleta que consiste em associar cada indivíduo a frações da área total de uma roleta. Nela cada uma destas frações são proporcionais à aptidão do indivíduo associado, fazendo com que haja uma probabilidade maior dos mais aptos serem escolhidos para reproduzirem(Kramer (2017)). Para a reprodução, o método adotado foi o two-point crossover que funciona gerando um novo indivíduo através da troca de uma cadeia de bits definida entre dois pontos de dois indivíduos(Ouerfelli and Dammak (2013)).

O Algoritmo dá início ao processo de evolução da solução, enviando dados de entrada para a simulação do sistema, que no nosso caso são os parâmetros PID. Assim o sistema retorna os erros que serão avaliados pelo algoritmo e que a partir disso irá gerar novos parâmetros. O que garante uma solução satisfatória é a repetição desse fluxo e a função avaliadora que procurará sempre minimizar o erro.

A figura 6 mostra o diagrama de blocos feito na ferramenta Simulink do software Matlab. Além de sua saída padrão, há três outras saídas que são os índices de desempenho: Integral Time Absolute Error (ITAE), Integral Absolute Error (IAE) e Integral Square Error (ISE). Indicados respectivamente pelas saídas 2,3 e 4 . Para a função de avaliação deste trabalho, foram testados os três índices.

$$
\begin{gathered}
I T A E=\int|e(t)| t^{2} d t \\
I A E=\int|e(t)| d t \\
I S E=\int e(t)^{2} d t
\end{gathered}
$$




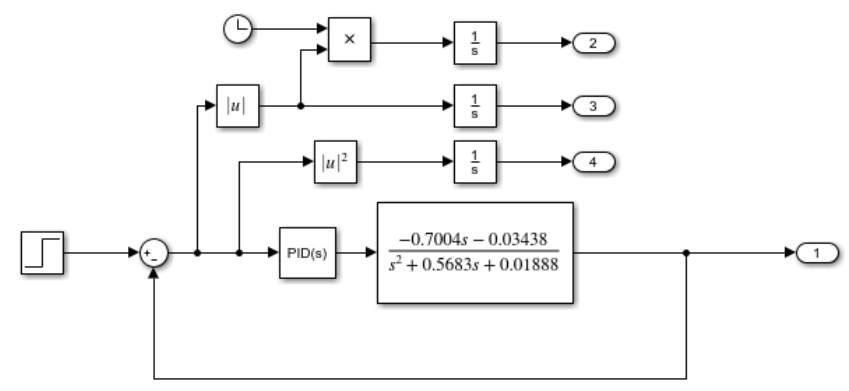

Figura 6. Simulação do sistema feita no Simulink.

Foi utilizada a toolbox de Algoritmo Genético do matlab e após 80 gerações foram encontrados os parâmetros para cada índice de desempenho exibidos na tabela 1. O gráfico da figura 7, mostra em cada geração, a sua média e o valor do indivíduo com o melhor índice, provando assim que a seleção dos mais aptos para a reprodução garantem novas gerações cada vez melhores.

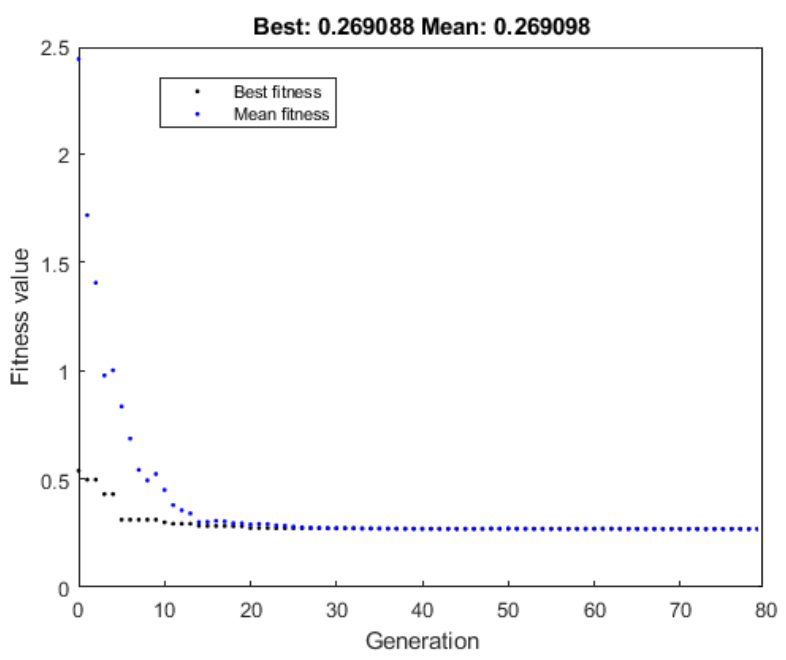

Figura 7. Gráfico exibindo a evolução para o índice ITAE.

Tabela 1. Resultados Parâmetros

\begin{tabular}{cccc} 
Índice & $\mathrm{P}$ & $\mathrm{I}$ & $\mathrm{D}$ \\
\hline ITAE & -2.9 & -1.5 & 0 \\
IAE & -2.9 & -1.7 & 0 \\
ISE & -1.2 & -1.9 & -2.7
\end{tabular}

Na figura 8 temos os dados do sistema ao rodar com os parâmetros obtidos pela otimização do IAE e ITAE. Para dois valores de amplitude diferente $120 \mathrm{~Hz}$ e $200 \mathrm{~Hz}$. Podese ver que os dois possuem comportamento similar, porém o referente à otimização do parâmetro ITAE apresentou uma melhor estabilidade. Já a figura 9 mostra o sistema rodando sob a otimização do parâmetro ISE. Como se pode notar, o sistema não operou de forma satisfatória. Foi então adotado a minimização do índice ITAE. Seus parâmetros gerados foram os que mostraram uma performance melhor quando aplicados ao chopper óptico, tanto em otimização do tempo de assentamento, quanto em garantir estabilidade.

Selecionado o PID que operou de forma melhor, foram feitos medidas do chopper funcionando para diversos set-

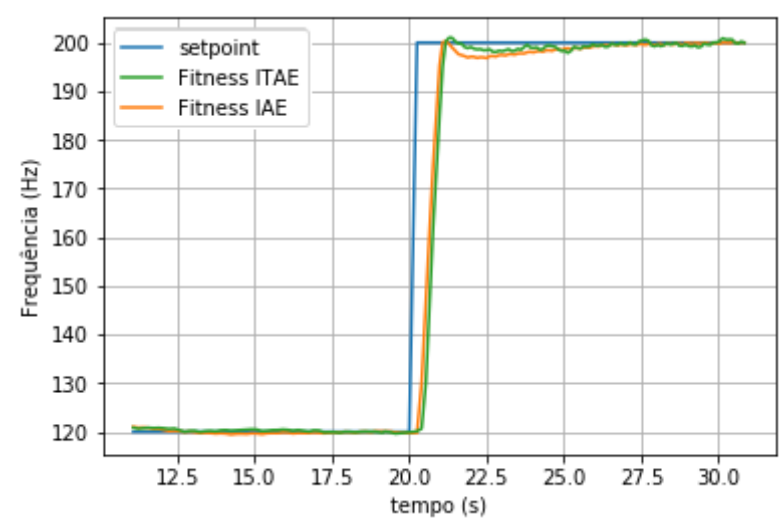

Figura 8. Gráfico do sistema usando os parâmetros obtidos minimizando o erro de ITAE e IAE.

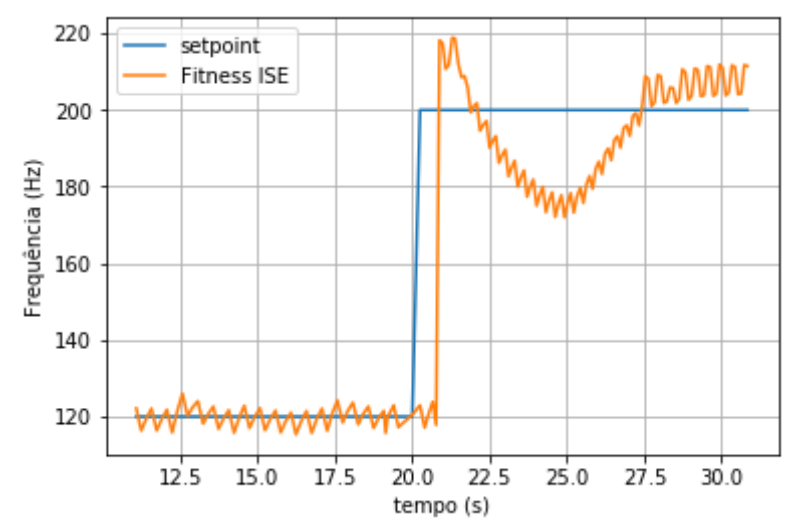

Figura 9. Gráfico do sistema para o parâmetro obtido minimizando o erro de ISE.

points diferentes (figura 10). Podemos notar ao comparar com a resposta ao degrau dada pelo matlab (figura 11) que o sistema testado no mundo real exibe uma diferença do simulado. Por exemplo, no simulado a resposta ao degrau unitário apresenta um tempo de assentamento menor. Ainda assim tem-se um desempenho aceitável para o sistema, ao entrar no estado estacionário o maior valor de erro(a diferença entre o valor medido e valor do setpoint dividido pelo valor do setpoint) foi de $0,21 \%$.

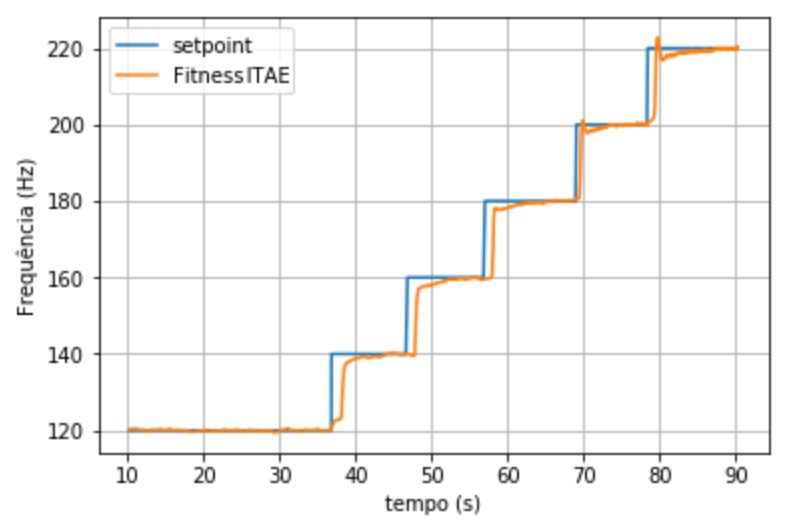

Figura 10. Gráfico do sistema para faixas de amplitudes diferentes. 


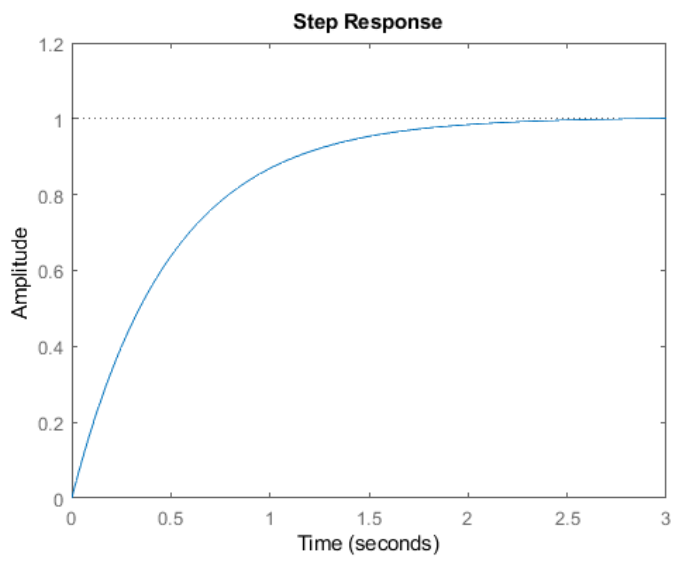

Figura 11. Resposta ao degrau pelo sistema utilizando os parâmetros obtidos pelo índice ITAE.

\section{DISCUSSÃO DOS RESULTADOS}

Diversos desafios foram apresentados a partir da proposta de se controlar a velocidade de rotação do disco de um chopper óptico sem um conhecimento prévio do modelo matemático. Dentre as diversas técnicas que podem ser implementadas nesta tarefa, as técnicas de identificação para gerar uma função de transferência e Algoritmo Genético para a obtenção dos parâmetros de controle mostraram resultados eficientes.

Existem diferenças específicas entre o modelo matemático e seu comportamento real, o que é observado ao comparar a simulação com os dados medidos. Porém, tais erros podem ser minimizados se escolhidas as técnicas certas. Diante disto, foi projetado um controle que atendeu à tarefa de controlar a frequência e garantir a sua estabilidade.

Ainda se deseja obter um tempo de resposta que seja mais eficiente, existe uma diferença significativa entre a simulação e o chopper operando. A presença de um overshooting antes da frequência entrar no regime estacionário também foi algo que não estava previsto pela resposta ao degrau da simulação.

Como possíveis trabalhos futuros, pode-se apontar uma estratégia de controle adaptativo a fim de se contornar possíveis instabilidades do sistema. Ou até mesmo a otimização do sistema por vias físicas, alterando sua estrutura de suporte ou material do disco dentado para garantir uma maior estabilidade.

\section{CONCLUSÃO}

Neste trabalho, construímos um chopper óptico de baixo custo e mostramos que a utilização de métodos de identificação e Algoritmo Genético são uma boa ferramenta para a sintonia dos valores de PID do sistema. A abordagem apresentada demonstra que não há necessidade de se ter um conhecimento matemático completo do sistema para se obter um bom controle, sendo este um método eficiente para se obter os parâmetros do sistema em um tempo reduzido.

Por fim, foi possível a construção de um instrumento funcional e com foco na redução de custos. O custo médio do projeto foi em torno de $\mathrm{R} \$ 110,00$, o que confirma ser uma excelente alternativa barata ao chopper profissional de U\$2.000,00.

\section{AGRADECIMENTOS}

Os autores agradecem pelo suporte financeiro fornecido pela Fundação Carlos Chagas de Amparo à Pesquisa do Estado do Rio de Janeiro(FAPERJ) e também pelo apoio da Universidade Federal do Rio de Janeiro(UFRJ) e Universidade do Estado do Rio de Janeiro(UERJ).

\section{REFERÊNCIAS}

Aguirre, L.A. (2007). Introdução à identificação de sistemas. Editora UFMG, Belo Horizonte.

Dorf, R. C Bishop, R.H. (2001). Sistemas de Controle Modernos. Addison Wesley Longman.

F. M. Amaral, Ricardo Tanscheit, M.A.C.P. (2009). Tuning pid controllers through genetic algorithms. Journal Information Sciences-Informatics and Computer Science, Intelligent Systems, 179, 1007-1018.

Fairweather, A., Foster, M., and Stone, D. (2011). Mls testing of vrla batteries using pseudo random binary sequences (prbs). World Electric Vehicle Journal, 4, 405-413. doi:10.3390/wevj4020405.

Farbman, D. and McCoy, C. (2016). Materials testing of 3d printed abs and pla samples to guide mechanical design. V002T01A015. doi:10.1115/MSEC2016-8668.

Kramer, O. (2017). Genetic Algorithm Essentials. Springer.

M. Penello, G., H. Pereira, P., Guerra, L., D. Pinto, L., Jakomin, R., T. Mourão, R., H. Degani, M., Maialle, M., Sivco, D., Gmachl, C., Pires, M., and L. Souza, P. (2019). Progress in symmetric and asymmetric superlattice quantum well infrared photodetectors. Annalen der Physik, 1800462. doi:10.1002/andp.201800462.

Ouerfelli, H. and Dammak, A. (2013). The genetic algorithm with two point crossover to solve the resourceconstrained project scheduling problems. 1-4. doi:10. 1109/ICMSAO.2013.6552686.

Zhang, J., Zhuang, J., Du, H., and Wang, S. (2009). Self-organizing genetic algorithm based tuning of pid controllers. Inf. Sci., 179, 1007-1018. doi:10.1016/j.ins. 2008.11.038. 